\title{
Associations among leaf developmental stability, canalization and phenotypic plasticity in response to population density in Abutilon theophrasti under contrasting soil conditions at different growth
} stages

\author{
Shu Wang ${ }^{1}$ and Dao-Wei Zhou ${ }^{2}$ \\ ${ }^{1}$ Guizhou University \\ ${ }^{2}$ Northeast Institute of Geography and Agroecology Chinese Academy of Sciences
}

November 24, 2021

\begin{abstract}
Most studies on animals have conducted comparative studies to deduce the possible relationships among developmental stability, canalization and phenotypic plasticity, there is a lack of direct evidence in plants, which should be better study materials. To investigate the correlations among developmental stability, canalization and plasticity in plants, we conducted a field experiment with Abutilon theophrasti, by subjected plants to three densities under infertile vs. fertile soil conditions, and measured leaf size, leaf fluctuating asymmetry (FA), and calculated coefficient of variation among leaves within individuals (CVleaf) and among individuals (CVin) and relative plasticity (PIrel) and its degree in leaf size at three growth stages, to analyze the responses of their correlations to density and how they may vary with soil conditions or growth stages. Results showed a decrease of FA, CVleaf and PIrel and an increase of CVin in leaf size, with increased density. In most cases, there were no correlations among these variables, but negative correlations between CVin and PIrel, positive correlations between FA and PIrel at high density and/or in fertile soil, in infertile soil. It suggested that higher FA may indicate the state of faster growth rather than an indicator of environmental stresses; there are correlations among developmental stability, canalization and plasticity, which may be complex, affected by other factors. The loss of developmental stability may be beneficial for plant response to environmental stresses, while decreased canalization can be either disadvantageous or advantageous, depending on that the size variation results from an increase or decrease of smaller individuals, and whether its correlations with other variables reflect beneficial or adverse environmental effects.
\end{abstract}

\section{Hosted file}

PD-FA-CV-P-soil 2021.11.doc available at https://authorea.com/users/358467/articles/546663associations-among-leaf-developmental-stability-canalization-and-phenotypic-plasticityin-response-to-population-density-in-abutilon-theophrasti-under-contrasting-soilconditions-at-different-growth-stages 